\title{
Optical principles of beam transport for relativistic electron cooling
}

\author{
A. Burov,* S. Nagaitsev, and A. Shemyakin \\ Fermi National Accelerator Laboratory, Batavia, Illinois 60510 \\ Ya. Derbenev \\ Physics Department, University of Michigan, Ann Arbor, Michigan 48109-1120
}

(Received 5 May 2000; published 26 September 2000)

\begin{abstract}
In conventional low energy electron coolers, the electron beam is immersed in a continuous solenoid, which provides a calm and tightly focused beam in a cooling section. While suitable for low energies, the continuity of the accompanying magnetic field is hardly realizable at relativistic energies. We consider the possibility of using an extended solenoid in the gun and the cooling section only, applying lumped focusing for the rest of the electron transport line.
\end{abstract}

PACS numbers: 29.27.Eg, 29.27.Fh

\section{INTRODUCTION}

Although electron cooling [1,2] has been a routine tool in many laboratories [3], its use has been restricted to low energy accelerators with the kinetic energy $<1 \mathrm{GeV} /$ nucleon, i.e., $<0.5 \mathrm{MeV}$ of electrons. Currently, there are two relativistic energy range electron cooling projects being developed: one is at Fermilab, for $8.9 \mathrm{GeV} / c$ antiprotons in the recycler ring [4], and the other is at DESY, for a $15-20 \mathrm{GeV} / c$ bunched proton beam in PETRA ring [5]. Traditional low energy electron cooling devices follow an original design of the cooler EPOKHA at the storage ring NAP-M [6] employing a continuous longitudinal magnetic field in the kilogauss range for the electron beam transport from the cathode through the cooling region to the collector; see Fig. 1.

The solenoidal field uniquely provides a focusing property, crucial for electron cooling: it allows one to confine tightly the electron beam while keeping its angular spread small. Although at higher energies the space charge and collective interaction effects become less destructive, the mentioned property of the solenoidal field makes it very beneficial. This was found to be true for the developed medium-relativistic projects $[4,5,7,8]$, but not only there. According to Refs. [9,10], the solenoidal field in the cooling section can be very advantageous at much higher energies as well. That is why a necessity of the longitudinal magnetic field in the cooling section is assumed in this paper. This does not mean that a possibility for effective cooling without the solenoidal field is totally denied; rather, such possibility, if found, would lie beyond the scope of this paper.

In principle, a continuous solenoid along the whole electron beam line suggested in Ref. [8] would be a good focusing option at any energy. Such a solution though is hardly compatible with the beam acceleration up to rela-

\footnotetext{
*Phone: (630) 840-8852. FAX: (630) 840-6311. Email address: burov@fnal.gov
}

tivistic energies and also with design advances (related to cooling of bunched beams) such as electron bunch decompression, incorporation of recirculator rings, etc. [5,10]. However, a different scale of electron energies under consideration allows one to modify this approach. Namely, lumped focusing can be used for the beam transport line with the idea of avoiding any coherent motion of the beam inside the cooling solenoid $[4,5,10]$.

A beam state required by the electron cooling is characterized by a high ratio between the beam size and the Larmor radius; this state is referred to as calm or magnetized. The transport line can include any separated optical elements such as solenoid lenses, dipoles, and quadrupoles. It is shown in this paper that a calm beam in the cooler requires certain matching between a magnetized electron gun and the cooler solenoid. A linear theory of matched four-dimensional optical transitions is presented which allows one to formulate properties of the transport line. The beam transformations are described in terms of the drift and the cyclotron degrees of freedom; the necessity of having them uncoupled is shown. For a beam born at a round cathode, it is proved that the cathode has to be properly magnetized. The possibility of transforming a ribbon electron beam in a storage ring into a calm beam in the cooler is discussed. An example of a conceptual design of relativistic electron cooling is shown assuming an electrostatic accelerator as a source of the electrons. In this device, only

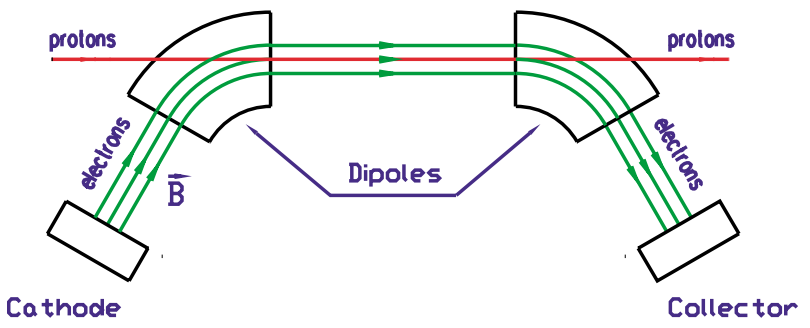

FIG. 1. (Color) Sketch of the NAP-M electron cooler EPOKHA. Electrons follow the magnetic field lines (green arrow lines) from the cathode to the collector. 
the gun, cooling section, and the collector are immersed in solenoids while the rest of the beam line has a lumped focusing.

\section{ANGULAR MOMENTUM-DOMINATED BEAM}

On entering or exiting the solenoid, the beam acquires a kick that changes its rotational state. Inside the cooling solenoid, the beam is required to be calm, i.e., not to have any angles in excess of the thermal ones (assumed to be negligible in this section). The above point is very important: the cooling rates are inversely proportional to a relative electron-ion velocity cubed; thus, any coherent angle above the thermal level dramatically depresses the cooling process. The questions under consideration are whether and how this requirement can be compatible with the lumped focusing scheme.

\section{A. Solenoids and acceleration intervals}

First, let the beam line consist of aligned solenoids and acceleration intervals only. For these straight and axially symmetric lattices, Busch's theorem states that the canonical angular momentum

$$
M=x p_{y}-y p_{x}=p r^{2} \theta^{\prime}-e \Phi(r, z) / 2 \pi c
$$

is conserved along any of the electron trajectories (see, e.g., $[11,12])$. Here, $x, y, p_{x}, p_{y}$ are the transverse Cartesian coordinates and their canonically conjugated momenta, $r, \theta, z$ are the cylindrical coordinates, the prime denotes a derivative along the axis $z, p=\gamma \beta m c$ is the total momentum, $\Phi(r, z)=2 \pi \int_{0}^{r} B(\tilde{r}) \tilde{r} d \tilde{r}$ is the magnetic flux inside a circle enclosed by the electron offset $r$, and $-e$ is the electron charge. The canonical angular momentum (CAM) of any electron is thus determined by its initial value, i.e., by its value at the cathode. Thus, the conservation of the CAM allows one to express an electron angular velocity at a given point of its trajectory in terms of the magnetic fluxes enclosed by this electron at this point and at the cathode. In the paraxial approximation, the magnetic field can be considered uniform over the beam cross section, which gives

$$
\theta^{\prime}=e\left(B r^{2}-B_{0} r_{0}^{2}\right) / 2 p c r^{2},
$$

with $B_{0}$ and $r_{0}$ the magnetic field and electron offset, respectively, at the cathode.

The single particle radial offset is described by the paraxial ray equation [12]

$$
\begin{aligned}
r^{\prime \prime}+\frac{\gamma^{\prime} r^{\prime}}{\beta^{2} \gamma}+\left(\frac{e B}{2 p c}\right)^{2} r-\left(\frac{e B_{0}}{2 p c}\right)^{2} \frac{r_{0}^{4}}{r^{3}} & \\
& =K \frac{r}{a^{2}}-\frac{\gamma^{\prime \prime} r}{2 \beta^{2} \gamma},
\end{aligned}
$$

where $a$ is the beam radius, $K=2 I / I_{0} \beta^{3} \gamma^{3}$ is the generalized perveance with $I$ standing for the current and $I_{0}=m c^{3} / e=17 \mathrm{kA}$. The right-hand side takes into account both the space charge and the external transverse electric field. The beam envelope $a=a(z)$ is found from Eq. (3) by the substitution $r=a, r_{0}=a_{0}$ with the initial conditions $a(0)=a_{o}, a^{\prime}(0)=0$. All the trajectories with $r^{\prime}(0)=0$ scale as $r(z)=r_{0} a(z) / a_{0}$.

Without the transverse electric fields, Eqs. (2) and (3) have an $r^{\prime}=0$ solution inside an extended solenoid of the cooler. This solution is realized if, after the entrance in the cooler,

$$
a^{\prime}=0, \quad B a^{2}=B_{0} a_{0}^{2} .
$$

At the exit of the gun solenoid, the beam acquires an azimuth velocity. During the transport, this velocity changes, partly transforming into the radial velocity. However, if the beam state at the entrance of the cooler is matched with its state at the cathode so that the conditions (4) are satisfied, the beam transverse velocities are finally canceled. Schematically, the beam transport with the matched entrance in the cooling solenoid is depicted in Fig. 2.

In the cooler, the space charge limits the minimal attainable angle, $\sqrt{a^{2} \theta^{\prime 2}+a^{\prime 2}}$, which cannot be zero. As follows from Eqs. (2) and (3), the angle is minimized when the cyclotron motion is not excited, $a^{\prime}=0$, and the angle is given by an azimuthal drift

$$
a \theta^{\prime}=2 I / \gamma^{2} \beta^{2} B a c .
$$

The drift angles can be neglected if they do not exceed the thermal angles of the cooled ions; this puts the lower boundary on the magnetic field in the cooling section.

With the drift neglected, the electrons do not have any transverse velocity inside the cooling solenoid provided that the matching conditions (4) are satisfied.

If the solenoid radius is much smaller than a period of the Larmor helix, the solenoid entrance can be considered as a thin boundary. In this case, the matching requirements (4) become explicit boundary conditions at the solenoid entrance. Note that, in the linear approximation, the matching is satisfied for every trajectory once it is satisfied for one of them.

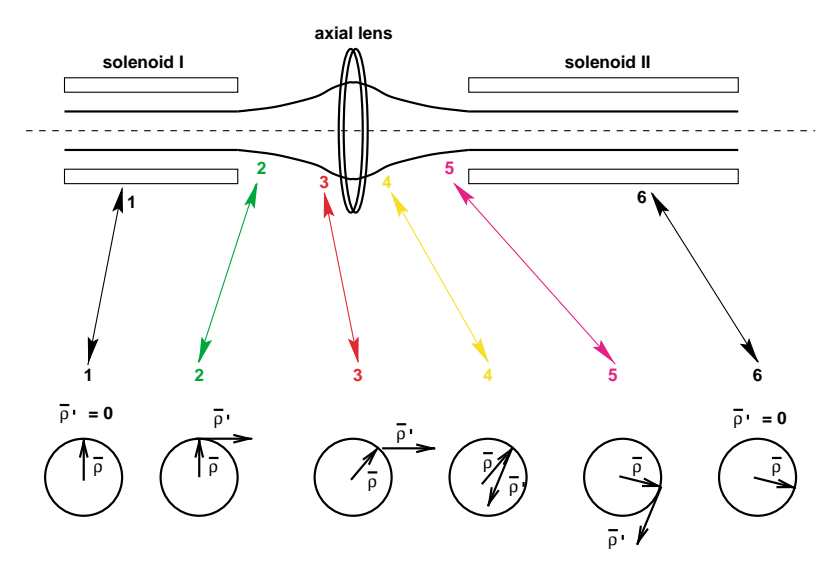

FIG. 2. (Color) Beam transport with matched entrance in the cooler. The beam envelope is depicted at the top. Transformation of electron coordinates $\vec{\rho}=(x, y)$ and velocities $\vec{\rho}^{\prime}=$ $\left(x^{\prime}, y^{\prime}\right)$ are shown at the bottom. 
In the radial equation (3), the last term on the left-hand side is determined by the inherited CAM $M=e B_{0} r_{0}^{2} / 2 c$. Radial dependence of this term allows one to treat the CAM as effective (unnormalized) emittance

$$
\varepsilon_{\mathrm{eff}}=M / p=\Phi / 2 \pi B \rho,
$$

with $B \rho=p c / e$. Then, this analogy leads to a concept of an effective beta function

$$
\beta_{\mathrm{eff}}=a^{2} / \varepsilon_{\mathrm{eff}}=\frac{2 \pi \gamma \beta e a^{2}}{r_{e} \Phi} .
$$

The effective beta function determines a required lensto-lens distance in the beam transport channel. For a conventional low-energy cooler, assuming the electron kinetic energy $E_{e}=25 \mathrm{keV}$, the magnetic flux $\Phi=1 \pi \mathrm{kG} \mathrm{cm}^{2}$, and the beam radius $a=1 \mathrm{~cm}$, the effective beta function is very small, $\beta_{\text {eff }}=1 \mathrm{~cm}$. It means that the accompanying magnetic field cannot be actually interrupted in this region of parameters. However, the situation changes for relativistic coolers where the accelerated beam is more rigid, and the magnetic flux can be significantly reduced.

For the Fermilab project, for instance, with the electron kinetic energy in the cooler $E_{e} \cong 4.3 \mathrm{MeV}$, the space charge limitation (5) allows for a rather small value for the magnetic flux: $\Phi=30 \pi \mathrm{G} \mathrm{cm}^{2}$ [4]. For the beam radius of $a=0.6 \mathrm{~cm}$, the accompanying magnetic field can be interrupted at early stages of the acceleration; for $\gamma \beta=2$ Eq. (7) already gives $\beta_{\text {eff }}=20 \mathrm{~cm}$.

When extracted from the magnetic field, the divergence of this cold and low space charge beam is determined by the inherited canonical angular momentum. Beams of this type can be referred to as an angular momentum dominated, as distinguished from emittance and space charge dominated beams.

The transport of the angular momentum dominated beam is not completely identical to that of the emittance dominated beams, as can be concluded from the envelope equation (3). The principal difference is that the CAMrelated angles are not random; once acquired, they can be effectively nulled out by a proper beam matching in the downstream solenoid, as sketched in Fig. 2. Because of the momentum spread of electrons, though, this extraction gets to be imperfect: electrons with different momenta have different phase advances. Note that the mismatch caused by the momentum spread increases with the magnetic field due to a growth of the phase advances and their spread; thus, from this point of view, a lower magnetic field is more beneficial. In this paper, that issue is not discussed in more detail; the electron momentum spread is supposed to be insignificant, which requires the phase-mismatch electron angles in the cooler to be smaller than the angles of the cooled ions.

\section{B. Bends}

Above, a straight transport line was considered. In practice, though, bending parts are normally inevitable. Thus, the next question is whether or not the bends could be compatible with this lumped focusing. Inside a dipole, the linear electron motion is conventionally described by the following set of equations:

$$
\begin{aligned}
x^{\prime \prime}+\frac{1-n}{\rho_{d}^{2}} x & =0, \quad y^{\prime \prime}+\frac{n}{\rho_{d}^{2}} y=0, \\
n & =-\frac{\rho_{d}}{B_{d}} \frac{\partial B_{d}}{\partial x},
\end{aligned}
$$

where $x$ and $y$ are the horizontal and vertical displacements from the ideal orbit, $\rho_{d}$ is a radius of curvature in the dipole magnetic field $B_{d}$ along the $y$ axis, and the parameter $n$ is conventionally referred to as the field index. Generally, these equations do not preserve the rotational symmetry of the beam in the transverse $x-y$ plane. However, for a specific case $n=1 / 2$ the equations are invariant under $x-y$ rotations, and the angular momentum $M=$ $p r^{2} \theta^{\prime}=p\left(x y^{\prime}-x^{\prime} y\right)$ is an integral of motion. Thus, for this specific field index, the bending parts are compatible with the lumped focusing scheme: the angular momentum conservation guarantees the same sufficient conditions for the calm beam in the cooler as for the straight transport line (4). For this invariant bending, the electron trajectory can be described in the polar coordinates

$$
r^{\prime \prime}+\frac{r}{2 \rho_{d}^{2}}-\left(\frac{M}{p}\right)^{2} \frac{1}{r^{3}}=0 .
$$

Thus, the transport line consisting of solenoids, drifts, and the index $1 / 2$ bending magnets would provide the calm beam inside the cooler if the matching conditions (4) were satisfied. From the optical point of view, all these elements are rotationally invariant; below, they are referred to as the invariant elements. Transport lines entirely based on the invariant elements can be called locally invariant; they are considered in the next section. The locally invariant lines have to be distinguished from another kind of a transport, which restores the rotation symmetry at the exit, but does not preserve it for the intermediate points of the trajectory. As a whole, such a kind of a transport is described by an invariant mapping, without being locally invariant. Such transport can be referred to as globally invariant. It can also be called block invariant if it relates to a part of the transport line.

In the succeeding section, the locally invariant transport is described in terms of $2 \times 2$ linear mapping. For these lines, the Courant-Snyder parameters are found and the matching conditions are reconsidered on a base of this approach.

\section{LOCALLY-INVARIANT MAPPING}

A linear mapping can be built in terms of the Cartesian coordinates $x$ and $y$. In the presence of solenoids, it is convenient to also introduce a rotating Larmor frame, $\hat{x}-\hat{y}$, as 


$$
\begin{aligned}
u & \equiv \hat{x}+i \hat{y}=(x+i y) e^{-i \chi}, \\
\chi^{\prime} & =\frac{1}{2} \frac{e B}{p c} \equiv \Omega / \gamma \beta c .
\end{aligned}
$$

Because of the symmetry, the equations of motion reduce to a single equation for the complex offset $u$ (see, e.g., [12]). This equation takes a most compact form when the beam frame time $\tau, d \tau=(\mathrm{m} / \mathrm{p}) d z$ is used as an independent variable instead of the longitudinal coordinate $z$ :

$$
\begin{aligned}
\ddot{u}+\hat{\Omega}^{2} u & =0, \\
\hat{\Omega}^{2} & =\Omega^{2}+\frac{1}{2}\left(\frac{e B_{d}}{m c}\right)^{2}-\frac{p^{2} K}{m^{2} a^{2}}+\frac{\gamma \gamma^{\prime \prime}}{2 c^{2}},
\end{aligned}
$$

which is the Mathieu-Hill equation describing the uncoupled betatron oscillations.

The solution of (11) can be presented in the conventional form

$$
\begin{aligned}
u(\tau) & =C_{+} \sqrt{\underline{\beta}} e^{i \phi}+C_{-} \sqrt{\underline{\beta}} e^{-i \phi} \equiv u_{+}+u_{-}, \\
\dot{\phi} & =1 / \underline{\beta},
\end{aligned}
$$

where $C_{+}, C_{-}$are two arbitrary complex constants, and the betatron function $\beta$ satisfies the following equation:

$$
2 \underline{\beta \ddot{\beta}}-\underline{\dot{\beta}}^{2}+4 \hat{\Omega}^{2} \underline{\beta}^{2}-4=0 .
$$

The solution of the equation of motion (11) can be written in a form that presents the constants $\left|C_{ \pm}\right|^{2}$ as the CourantSnyder invariants,

$$
\left|C_{ \pm}\right|^{2}=\frac{1}{4 \underline{\beta}}|u(1 \pm i \underline{\dot{\beta}} / 2) \mp i \underline{\beta} \dot{u}|^{2},
$$

which can be also expressed as

$$
\begin{aligned}
\left|C_{ \pm}\right|^{2}=\frac{1}{4 \underline{\beta}} & {\left[(1 \mp \underline{\beta} \Omega)^{2} r^{2}+\dot{\beta}^{2} r^{2} / 4\right.} \\
& +\underline{\beta}^{2}\left(\dot{r}^{2}+r^{2} \dot{\theta}^{2}\right) \\
& \left. \pm \underline{2} \underline{\beta}(1 \mp \underline{\beta} \Omega) r^{2} \dot{\theta}-\underline{\beta \dot{\beta}} r \dot{r}\right] .
\end{aligned}
$$

From here, it follows that the two squared amplitudes $\left|C_{ \pm}\right|^{2}$ are related to each other by means of the CAM:

$$
\left|C_{+}\right|^{2}-\left|C_{-}\right|^{2}=M / m \text {. }
$$

To be complete, the presentation [(12) and (13)] requires the initial conditions for the beta function, $\underline{\beta}(0), \dot{\beta}(0)$. Generally speaking, these initial conditions can be arbitrarily chosen for transport lines. However, in the case under study the starting point $\tau=0$ corresponds to a surface of the magnetized cathode, and this determines a natural choice for the initial beta function. There are no transverse fields at the cathode, $\hat{\Omega}_{0}=\Omega_{0}$, and a trajectory with zero initial transverse velocities, $\dot{r}=0, r \dot{\theta}=0$, does not have initial cyclotron amplitude. It is convenient to identify this particular trajectory with a pure "minus" solution, with $C_{+}=0$ for it, which is realized by a choice of

$$
\underline{\beta}(0)=1 / \Omega_{0}, \quad \underline{\dot{\beta}}(0)=0 .
$$

With this choice, the amplitude $C_{-}$is determined by an initial offset, while $C_{+}$is a function of the initial transverse velocity. So the minus solution relates to the position of the Larmor center inside the gun solenoid, and the "plus" solution describes the cyclotron excitation there. At the cathode, they satisfy the boundary conditions

$$
\left.\dot{u}_{ \pm}\right|_{0}= \pm\left. i \Omega_{0} u_{ \pm}\right|_{0} .
$$

As shown in Sec. IV, these plus and minus solutions can be treated as two canonical degrees of freedom, referred to as a drift and a cyclotron motion.

The drift and the cyclotron solutions can also be considered inside the cooling solenoid, where $\hat{\Omega}=\hat{\Omega}_{f}=$ const. These particular solutions satisfy conditions simiar to (18):

$$
\left.\dot{u}_{ \pm}\right|_{f}= \pm\left. i \hat{\Omega}_{f} u_{ \pm}\right|_{0} .
$$

\section{A. Matched mapping}

Solutions of the Mathieu-Hill equation (11) can also be presented in terms of a transformation with a real $2 \times 2$ matrix, $A(\tau)$ :

$$
\left(\begin{array}{l}
u \\
\dot{u}
\end{array}\right)_{\tau}=\mathrm{A}(\tau)\left(\begin{array}{l}
u \\
\dot{u}
\end{array}\right)_{0}, \quad|\mathrm{~A}(\tau)|=1 .
$$

When an initially calm beam is finally transformed into a calm state again, it means that the cyclotron mode is not excited either initially or finally or

$$
\dot{u}_{0}=-i \Omega_{0} u_{0}, \quad \dot{u}_{f}=-i \hat{\Omega}_{f} u_{f} .
$$

This matching imposes the following conditions on the transformation matrix A (20):

$$
\frac{A_{22}}{A_{11}}=\frac{\hat{\Omega}_{f}}{\Omega_{0}} ; \quad \frac{A_{21}}{A_{12}}=-\hat{\Omega}_{f} \Omega .
$$

From here, the matrix $A$ can be parameterized as

$$
\mathrm{A}=\left(\begin{array}{cc}
\sqrt{\frac{\Omega_{0}}{\hat{\Omega}_{f}}} \cos \psi & \frac{1}{\sqrt{\hat{\Omega}_{f} \Omega_{0}}} \sin \psi \\
-\sqrt{\hat{\Omega}_{f} \Omega_{0}} \sin \psi & \frac{\hat{\Omega}_{f}}{\Omega_{0}} \cos \psi
\end{array}\right),
$$

with a necessary condition $\hat{\Omega}_{f} \Omega_{0}>0$. The single free parameter, a phase $\psi$, is determined by all the involved optic elements, with $\dot{\psi}=\hat{\Omega}_{f}$ inside the cooling solenoid.

The presentation (23) can also be obtained in a different manner. The matrix of transformation for the MathieuHill equation has a conventional expression in terms of the initial and final values of the betatron function and its derivative (see, e.g., [13]). The initial values of the beta function, and its derivative are considered above: $\underline{\beta}_{0}=1 / \Omega_{0}, \underline{\beta}_{0}=0$. If the cyclotron mode is not excited in the cooler, similar conditions are fulfilled there: 
$\underline{\beta}_{f}=1 / \hat{\Omega}_{f}, \underline{\dot{\beta}}_{f}=0$. With these initial and final values of the betatron function, the above presentation of the matrix $A$ (23) follows.

It results from (21) and (23) that, if the cyclotron mode is not finally excited, then the initial and final beam sizes are matched:

$$
\left(\hat{\Omega} r^{2}\right)_{f}=\left(\Omega r^{2}\right)_{0} .
$$

A difference between this form of the matching condition and the "magnetic flux law" (4) reflects a beam drift rotation under the space charge effect. It is shown below that the corrected matching condition (24) expresses a restoration of the action related to the drift degree of freedom.

The equation of motion (11) corresponds to the Hamiltonian

$H\left(\hat{x}, \hat{p}_{x}, \hat{y}, \hat{p}_{y}\right)=\frac{\hat{\Omega}^{2} \hat{x}^{2}}{2}+\frac{\hat{p}_{x}^{2}}{2}+\frac{\hat{\Omega}^{2} \hat{y}^{2}}{2}+\frac{\hat{p}_{y}^{2}}{2}$,

with $\hat{p}_{x}, \hat{p}_{y}$ being canonical momenta conjugated to the variables $\hat{x}, \hat{y}$. For that part of the trajectory where $\hat{\Omega}=$ const,

$$
\hat{J}_{x}=\frac{\hat{\Omega} \hat{x}^{2}}{2}+\frac{\hat{p}_{x}^{2}}{2 \hat{\Omega}}, \quad \hat{J}_{y}=\frac{\hat{\Omega} \hat{y}^{2}}{2}+\frac{\hat{p}_{y}^{2}}{2 \hat{\Omega}}
$$

are the corresponding action variables. For the pure drift motion, both actions are equal:

$$
\hat{J}_{x}=\hat{J}_{y}=\hat{\Omega} r^{2} / 2 .
$$

It can also be seen that the actions are preserved under the transformation A. Generally, the actions do not vary when the system parameters change adiabatically. Although the beam transport is not supposed to be adiabatic, the actions are still preserved here. This property of mapping A can be interpreted in a general way. The conditions (21) express a requirement for the mapping not to mix the two modes of the motion. Keeping the modes uncoupled is also a general property of the adiabatic motion. That is why it is not a surprise that the action preservation is guaranteed for both cases.

As a curious fact, it can be noted that the equation of motion (11) can be associated with a complex Hamiltonian

$$
H\left(u, p_{u}\right)=\frac{\hat{\Omega}^{2} u^{2}}{2}+\frac{p_{u}^{2}}{2} .
$$

Then, the complex action

$$
\hat{J}_{u}=\frac{\hat{\Omega} u^{2}}{2}+\frac{p_{u}^{2}}{2 \hat{\Omega}}
$$

is also conserved: initially and finally $J_{u}=0$.

\section{B. Temperature transformation for a matched transport}

Above, the transformation matrix A (23) was found from the condition of the drift-to-drift transition (21). Since the matrix is invariant with respect to a common sign change of $\Omega_{0}$ and $\hat{\Omega}_{f}$, it allows one to conclude that matching of the drift component leads automatically to identical matching of the cyclotron component:

$$
\left(\hat{\Omega}\left|u_{ \pm}\right|^{2}\right)_{f}=\left(\Omega\left|u_{ \pm}\right|^{2}\right)_{0} .
$$

For the drift mode (+), it gives the conditions (24), while for the cyclotron mode $(-)$ it can be rewritten in terms of the transverse temperature $T_{\perp}$ :

$$
\left(\frac{T_{\perp}}{\hat{\Omega}}\right)_{f}=\left(\frac{T_{\perp}}{\Omega}\right)_{0} .
$$

It is shown below that the relationships (30) may be interpreted as a preservation of both drift and cyclotron actions when these modes do not transfer to each other.

\section{MATCHING WITH NONINVARIANT OPTIC ELEMENTS}

The analysis above was related to the locally invariant transportation, i.e., based on such optically invariant elements as the solenoids and dipoles with the index $1 / 2$. However, with increasing the electron energy, the quadrupoles can be more suitable than the solenoid lenses for the beam transport. Also, the conventional uniformfield dipoles may look more preferable than the $1 / 2$ index dipoles from a technical point of view. Consequently, a question appears as to whether such optical elements as conventional dipoles or quadrupoles are compatible with the requirement to have a calm beam in the cooling section.

\section{A. Uncoupled transformation}

In this section, a general form of the transformation matrix is found. An optical transition between the magnetized cathode and the cooler can be treated in terms of the canonically conjugated pairs. Let $\vec{\rho}=(x, y)$ be the transverse Cartesian coordinate and $\vec{p}_{\perp}=\left(p_{x}, p_{y}\right)=\vec{k}_{\perp}-$ ${ }_{c} \vec{A}_{\perp}$ be the canonically conjugated momentum. Here $\vec{k}_{\perp}=\gamma m \vec{v}_{\perp}$ is the kinetic momentum and $\vec{A}_{\perp}=\frac{1}{2} \vec{B} \times$ $\vec{\rho}$ is the vector potential in the solenoid. The transformation of a particle state

$$
\mathbf{x}=\left(\begin{array}{c}
x \\
p_{x} \\
y \\
p_{y}
\end{array}\right)
$$

is expressed as $\mathbf{x}_{f}=\mathcal{T} \mathbf{x}_{0}$ with a symplectic $4 \times 4$ matrix $\mathcal{T}$. The mapping symplecticity can be expressed as invariance of the Poisson brackets

$$
\{f, g\} \equiv \sum_{i=x, y}\left(\frac{\partial f}{\partial p_{i}} \frac{\partial g}{\partial \rho_{i}}-\frac{\partial g}{\partial p_{i}} \frac{\partial f}{\partial \rho_{i}}\right)
$$

under this transformation for any two functions $f=$ $f(\vec{p}, \vec{\rho}), g=g(\vec{p}, \vec{\rho})$ (see, e.g., $[14,15]$ ). In particular, there are only two nonzero Poisson brackets between the components of the state vector $\mathbf{x}_{f}$ as functions of the components of the initial state $\mathbf{x}_{0}$ : 


$$
\left\{p_{x}, x\right\}=\left\{p_{y}, y\right\}=1,
$$

while the rest of the four brackets are equal to zero.

For a given point $\mathbf{x}$ in the $4 \mathrm{D}$ phase space, the transverse kinetic momentum $\vec{k}$ and the position $\vec{d}$ of the Larmor center are expressed as

$$
\begin{aligned}
\vec{k}_{\perp} & =\vec{p}_{\perp}+\frac{e}{2 c} \vec{B} \times \vec{\rho}, \\
\vec{d} & =\vec{\rho}-\vec{\rho}_{L}=\frac{1}{2} \vec{\rho}-\frac{c}{e} \frac{\vec{p} \times \vec{B}}{B^{2}},
\end{aligned}
$$

where the vector $\vec{\rho}_{L}=c \vec{k} \times \vec{B} / e B^{2}$ describes the position on the Larmor circle relative to its center. The relationships (34) can be considered as a transformation from the canonical pair $\vec{\rho}$ and $\vec{p}$ to the new variables $\vec{d}$ and $\vec{k}$. The nontrivial feature of this transformation is that the Poisson's brackets between $\vec{k}$ and $\vec{d}$ are equal to zero, while

$$
\left\{k_{x}, k_{y}\right\}=-\frac{e B}{c}, \quad\left\{d_{x}, d_{y}\right\}=\frac{c}{e B} .
$$

Therefore, the normalized variables

$\left(\begin{array}{l}\kappa_{1} \\ \kappa_{2}\end{array}\right)=\sqrt{\frac{c}{e B}}\left(\begin{array}{l}k_{y} \\ k_{x}\end{array}\right)$ and $\left(\begin{array}{l}\xi_{1} \\ \xi_{2}\end{array}\right)=\sqrt{\frac{e B}{c}}\left(\begin{array}{l}d_{x} \\ d_{y}\end{array}\right)$

compose new canonical pairs. The action and phase variables related to these pairs can be introduced as well:

$$
\left(\begin{array}{l}
\xi_{1} \\
\xi_{2}
\end{array}\right)=\sqrt{2 J_{D}}\left(\begin{array}{c}
\cos \psi_{D} \\
\sin \psi_{D}
\end{array}\right), \quad\left(\begin{array}{l}
\kappa_{1} \\
\kappa_{2}
\end{array}\right)=\sqrt{2 J_{C}}\left(\begin{array}{c}
\cos \psi_{C} \\
\sin \psi_{C}
\end{array}\right),
$$

with

$$
\begin{aligned}
& J_{D}=\left(\xi_{1}^{2}+\xi_{2}^{2}\right) / 2 \equiv \xi^{2} / 2=\frac{e B}{2 c} d^{2}, \\
& J_{C}=\left(\kappa_{1}^{2}+\kappa_{2}^{2}\right) / 2 \equiv \kappa^{2} / 2=\frac{c}{2 e B} k^{2} .
\end{aligned}
$$

[A canonical transformation similar to (34)-(38) is mentioned in Ref. [14], p. 432.] In terms of these new variables, the CAM is expressed in a very compact way:

$$
M=\frac{e B}{2 c}\left(d^{2}-\rho_{L}^{2}\right)=\frac{\xi^{2}-\kappa^{2}}{2}=J_{D}-J_{C} .
$$

This canonical transformation can be presented as

$$
\hat{\mathbf{x}} \equiv\left(\begin{array}{c}
\kappa_{1} \\
\kappa_{2} \\
\xi_{1} \\
\xi_{2}
\end{array}\right)=\mathcal{B}\left(\begin{array}{c}
x \\
p_{x} \\
y \\
p_{y}
\end{array}\right) \equiv \mathcal{B} \mathbf{x}
$$

with a symplectic $4 \times 4$ matrix $\mathcal{B}$, which can be composed using (34) and (36). Finally, the transformation between the two solenoids can be rewritten as

$$
\hat{\mathbf{x}}_{f}=\hat{\mathcal{T}} \hat{\mathbf{x}}_{i}
$$

with a new symplectic matrix

$$
\hat{\mathcal{T}}=\mathcal{B}_{f} \mathcal{T} \mathcal{B}_{0}^{-1}
$$

where the matrices $\mathcal{B}_{0}$ and $\mathcal{B}_{f}$ belong to the initial (electron gun) and final (cooling section) solenoids, respectively.

The $4 \times 4$ matrix $\hat{\mathcal{T}}$ can be presented in a block form as

$$
\hat{\mathcal{T}} \equiv\left(\begin{array}{ll}
(\mathrm{CC}) & (\mathrm{CD}) \\
(\mathrm{DC}) & (\mathrm{DD})
\end{array}\right)
$$

In the initial state (at the cathode), the beam diameter largely exceeds a characteristic Larmor radii of particles. It can be expressed as a high initial excitation of the drift degree of freedom in comparison with the cyclotron one. To minimize the cyclotron motion in the cooling solenoid, any influence from the drift degree of freedom has to be avoided. In other words, the beam transport should be designed in a way that $2 \times 2$ block (CD) vanishes. So the Poisson bracket $\left\{\kappa_{1}, \kappa_{2}\right\}=1$ is determined by the matrix (CC) only; therefore,

$$
\left(\begin{array}{l}
\kappa_{1} \\
\kappa_{2}
\end{array}\right)_{f}=(\mathrm{CC})\left(\begin{array}{l}
\kappa_{1} \\
\kappa_{2}
\end{array}\right)_{0}, \quad|(\mathrm{CC})|=1 .
$$

Because $|\hat{\mathcal{T}}|=1,|(D D)|=1$. Finally, it can be shown that the block (DC) also vanishes; it follows from the fact that all the Poisson brackets $\left\{\kappa_{i}, \xi_{j}\right\}=0$. As a result, the transformation of the drift component reduces to

$$
\left(\begin{array}{l}
\xi_{1} \\
\xi_{2}
\end{array}\right)_{f}=(\mathrm{DD})\left(\begin{array}{l}
\xi_{1} \\
\xi_{2}
\end{array}\right)_{0}, \quad|(\mathrm{DD})|=1 .
$$

The obtained block-diagonal form of the transformation

$$
\hat{\mathcal{T}}=\left(\begin{array}{cc}
(\mathrm{CC}) & 0 \\
0 & (\mathrm{DD})
\end{array}\right)
$$

shows that the mutual uncoupling of the drift and cyclotron degrees of freedom is necessary and sufficient for having a calm beam in the cooling section. Under this uncoupled transformation, both the drift and the cyclotron rms emittances,

$$
\begin{aligned}
& \epsilon_{D}=\sqrt{\left\langle\xi_{1}^{2}\right\rangle\left\langle\xi_{2}^{2}\right\rangle-\left\langle\xi_{1} \xi_{2}\right\rangle^{2}}, \\
& \epsilon_{C}=\sqrt{\left\langle\kappa_{1}^{2}\right\rangle\left\langle\kappa_{2}^{2}\right\rangle-\left\langle\kappa_{1} \kappa_{2}\right\rangle^{2}},
\end{aligned}
$$

are preserved; the brackets \langle\rangle stand for the ensemble averaging.

If the matched mapping is (globally) rotation invariant, then the matrices (CC) and(DD) are also invariant. The group of rotationally invariant $2 \times 2$ transformations is the group of rotations itself, so

$$
\begin{aligned}
& (\mathrm{CC})=\left(\begin{array}{cc}
\cos \psi_{C} & \sin \psi_{C} \\
-\sin \psi_{C} & \cos \psi_{C}
\end{array}\right), \\
& (\mathrm{DD})=\left(\begin{array}{cc}
\cos \psi_{D} & \sin \psi_{D} \\
-\sin \psi_{D} & \cos \psi_{D}
\end{array}\right),
\end{aligned}
$$

with the two phases $\psi_{C}, \psi_{D}$ as free parameters. In this case, the actions $\xi^{2} / 2=J_{D}$ and $\kappa^{2} / 2=J_{C}$ are not changed. This again leads to the relations (24) and (31) 
found above for the locally invariant transport. Here, these relations reveal themselves as conditions of the actions preservation; also, they express the restoration of the emittances (46). Indeed, in this case the cross averages in (46) vanish, which results in

$$
\begin{aligned}
& \epsilon_{D}=\left\langle J_{D}\right\rangle=e B / c\left\langle d^{2} / 2\right\rangle, \\
& \epsilon_{C}=\left\langle J_{C}\right\rangle=c / e B\left\langle k^{2} / 2\right\rangle .
\end{aligned}
$$

Because of the decoupling of the drift and cyclotron degrees of freedom, the $4 \mathrm{D}$ emittance follows as

$$
\epsilon=\epsilon_{D} \epsilon_{C}=d^{2} k^{2} / 4 .
$$

The last result can be found in a different way. Generally, the $4 \mathrm{D}$ emittance is calculated by means of the $4 \times 4$ correlation matrix $\Sigma_{i k}=\left\langle\mathbf{x}_{i} \mathbf{x}_{k}\right\rangle$, as $\epsilon=\sqrt{|\Sigma|}$. For arbitrary axially symmetric beams, this results in [16]

$$
\epsilon=\left(\left\langle r^{2}\right\rangle\left\langle k^{2}\right\rangle-\left\langle r k_{r}\right\rangle^{2}-\left\langle r k_{\theta}\right\rangle^{2}\right) / 4,
$$

with $k_{r}$ and $k_{\theta}$ being the radial and the axial components of the kinetic momentum. For the matched beam, this expression can be presented in terms of the drift and cyclotron variables (34). When vanishing correlations between the drift and the cyclotron degrees of freedom are taken into account, $\left\langle d_{i} k_{j}\right\rangle=0$, the previous result (50) follows.

Normally, the hadron beams have equal transverse emittances. Then, the axial symmetry of the transverse momentum distribution of the hadron beam in the cooler is beneficial for the cooling process. Thus, the optimal cross section of the hadron beam is also axially symmetric there. That is why a round shape of the electron beam in the cooling section is also optimal. Taking into account that a round shape of the cathode is also preferable, it leads to a conclusion that in the optimum the drift matrix (DD) is rotationally invariant (48). If the cyclotron motion (temperature) can be neglected in the initial state, no requirements are imposed on the matrix (CC), so it can be an arbitrary matrix with unit determinant. In an opposite case, the electron cyclotron motion depresses the cooling rates. This depression is minimal for rotationally invariant beam distribution in the cyclotron phase space. For the 2D transformations (CC), it means that the transformation itself has to be invariant (47) in this case. The total transformation (45) is rotation invariant if and only if the invariance of both of its components (DD) and (CC) is provided.

\section{B. Invariant matrices}

According to the above description, the decoupled invariant beam transformations preserve the CAM. Thus, it would be convenient for the electron transport line to consist of the invariant blocks, i.e., groups of the optic elements described by the CAM-preserving matrices. A group of such linear mappings was considered by Pozdeev [17] and Perevedentsev [18] and was discussed in [19]. It was proved that all CAM-preserving matrices are described by the following $2 \times 2$ block-diagonal form:

$$
\mathcal{T}=\mathcal{U}(\psi)\left(\begin{array}{ll}
\mathrm{T} & 0 \\
0 & \mathrm{~T}
\end{array}\right) .
$$

Here $\mathcal{U}(\psi)$ is a $4 \mathrm{D}$ rotation matrix providing separated rotations in the coordinate and momentum subspaces by the same angle $\psi$, and $\mathrm{T}$ is an arbitrary $2 \times 2$ matrix with $|\mathrm{T}|=1$ required by the phase volume preservation. Note that the group (52) can also be described as a group of rotation invariant transformations because of $\mathcal{U}^{T} \mathcal{T} \mathcal{U}=$ $\mathcal{T}$, where the superscript $T$ stands for transposing. This condition is equivalent to a commutation of the matrices $\mathcal{T}$ and $\mathcal{U}$ due to the rotation unitarity, $\mathcal{U}^{-1}=\mathcal{U}^{T}$. It follows that the mapping (52) transforms any round beam distribution into round again. Note that matrices

$$
\mathcal{T}=\mathcal{U}(\psi)\left(\begin{array}{cc}
\mathrm{T} & 0 \\
0 & -\mathrm{T}
\end{array}\right)
$$

also transform any round beam into round again, but they change the sign of the CAM. It can be shown that apart from the sets (52) and (53) there are no other matrices transforming any round beam into round again.

Without coupling of the transverse degrees of freedom $(\psi=0)$, the invariance requires for $x$ and $y$ matrices to be identical, which constitutes three independent conditions. Thus, two variable quadruples with one variable drift (or three variable quadruples) are sufficient to transmute any initial mapping into an invariant one.

\section{NONINVARIANT TRANSFORMATIONS}

A transport scheme above requires the cathode immersed in a proper solenoidal field. A question arises as to whether the magnetic field at the cathode is really inevitable. If one assumes the (global) rotation invariance for the transport mapping, the answer is clearly positive: this immediately follows from the CAM conservation for these transformations. But is it still possible to eliminate this field for some noninvariant transport?

Note that a noninvariant mapping can transform a particular calm and round beam into calm and round state again. An example of this was actually shown in the previous section. It was pointed out there that the cyclotron motion is not excited by the decoupled transformations. If this motion were not excited initially, an invariant drift transformation (DD) is sufficient to have final beam round when the initial beam was round too. Invariance of the cyclotron matrix (CC) is not required here; this matrix can be arbitrary. In this case, the total transformation $\mathcal{T}$ is not invariant, but it still provides a round-to-round beam transformation for a particularly initial state.

A relationship between the group of transformations preserving the symmetry of a particular beam state and the group of invariant transformations, which preserves the symmetry of any beam state, can be considered in a more general way. Let $\mathbf{x}$ be a vector in the $4 \mathrm{D}$ phase space. A particular symmetric beam state can be constructed as a set of points obtained from this vector by rotations, $\mathcal{U}(\psi) \mathbf{x}$. 
A transformation $\mathcal{T}$ preserves the symmetry for this particular state when the image of the rotated vector equals the rotated image of the initial vector:

$$
\mathcal{T} \mathcal{U}(\psi) \mathbf{x}=\mathcal{U}(\psi) \mathcal{T} \mathbf{x}
$$

which can also be formulated as a commutation of the mapping $\mathcal{T}$ and the rotations $\mathcal{U}(\psi)$ on the particular vector $\mathbf{x}$. The transformations (54) are not invariant generally: their commutators with the rotations do not give zero when applied to an arbitrary phase space vector, as the invariant transformations do. Instead, these commutators give zero only at the particular subspace or phase space projection related to the vector $\mathbf{x}$. That is why these transformations can be called projective invariant.

To describe the projective-invariant group in more detail, the one-parametric group of rotations can be expanded as

$$
\mathcal{U}(\psi)=I \cos (\psi)+\tilde{I} \sin (\psi),
$$

with $I$ as the identity and $\tilde{I}$ as the $90^{\circ}$ turn $4 \times 4$ matrices. After this substitution, the projective invariance condition (54) reduces to

$$
\mathcal{T} \tilde{I} \mathbf{x}=\tilde{I} \mathcal{T} \mathbf{x},
$$

where the rotation angle $\psi$ does not enter any more. For the given vector $\mathbf{x}$, this gives four additional independent equations on the matrix elements of $\mathcal{T}$. Remembering that an arbitrary symplectic $4 \times 4$ matrix has $16-3-2-$ $1=10$ free parameters, it follows that the $4 \mathrm{D}$ projectiveinvariant group has $10-4=6$ free parameters, two parameters more than its subset, the group of invariant transformations (52) and (53). Thus, the projectiveinvariant group is significantly wider than its subset, the invariant group. Therefore, a question of CAM preservation for it is not trivial.

\section{A. Generalized Busch's theorem}

Thus, the problem can be rephrased in the following manner: do the projective-invariant mappings preserve the CAM? In other words, assuming the beam to be round at the cathode, does it have to be properly magnetized (4) to get the beam quiet and round inside the downstream solenoid? Remember that the mapping invariance is not employed in this section.

A positive answer to this question follows from the generalized Busch's theorem [20]. The theorem states that, for a hydrodynamic, or laminar, beam transported by means of arbitrary static electric and magnetic fields, the contour integral

$$
\oint_{\Gamma} \vec{p} \vec{d} l=\oint_{\Gamma} \vec{k} \vec{d} l-e \Phi / c
$$

is conserved. Here the contour $\Gamma$ bounds an arbitrary tube of trajectories in the 3D coordinate space $x, y, z$. If the initial and final beam states are rotationally invariant, the contour $\Gamma$ is a circumference in the transverse plane, and the CAM preservation follows. Note that the field linearity is not required here.

Below, this theorem is extended from the electrostatic and magnetostatic fields to arbitrary Hamiltonian systems. This extension, however, requires the assumption of the linearity of the transformation. Thus, the statement to be proved claims the following: if a particular round beam is transformed by a symplectic linear mapping into a round state again, the CAM of every particle is restored. Note that the beam is not supposed to be laminar here.

A property of the symplectic transformations to conserve skew-scalar products is used here (see, e.g., [15]). The skew-scalar product of two vectors in the $4 \mathrm{D}$ transverse phase space $\mathbf{x}_{1}=\left(x_{1}, p_{x 1}, y_{1}, p_{y 1}\right)$ and $\mathbf{x}_{2}=\left(x_{2}, p_{x 2}, y_{2}, p_{y 2}\right)$ is an antisymmetric bilinear form $\left[\mathbf{x}_{1}, \mathbf{x}_{2}\right]$. Expressed in terms of the usual scalar product, it can be written as $\left[\mathbf{x}_{1}, \mathbf{x}_{2}\right]=\left(\mathbf{x}_{1}, \mathbf{S} \mathbf{x}_{2}\right)$ with $S$ as a rotation by $90^{\circ}$ in each of the phase planes, or

$$
\left[\mathbf{x}_{1}, \mathbf{x}_{2}\right]=-x_{1} p_{x 2}-y_{1} p_{y 2}+x_{2} p_{x 1}+y_{2} p_{y 1} .
$$

Let $\mathbf{x}_{1 i}$ and $\mathbf{x}_{2 i}$ be two arbitrary vectors of the initial state finally transformed into $\mathbf{x}_{1 f}$ and $\mathbf{x}_{2 f}$. Because of the symplecticity,

$$
\left[\mathbf{x}_{1 i}, \mathbf{x}_{2 i}\right]=\left[\mathbf{x}_{1 f}, \mathbf{x}_{2 f}\right]
$$

for any choice of $\mathbf{x}_{1}$ and $\mathbf{x}_{2}$. It can be seen that the angles between their 2D $x-y$ components are conserved by the transformation. This property is an obvious consequence of the rotation invariance of both states; without it, there would be an angular asymmetry of the final beam density distribution. However, the sign of this angle can be changed that would not contradict the angular symmetry of the final state. The two initial vectors can be taken as 2D orthogonal:

$$
\begin{aligned}
& \mathbf{x}_{1 i}=\left(r_{i}, p_{i r}, 0, p_{i t}\right), \\
& \mathbf{x}_{2 i}=\tilde{\mathbf{x}}_{1 i} \equiv\left(0,-p_{i t}, r_{i}, p_{i r}\right),
\end{aligned}
$$

having the angular momentum $M_{i}=r_{i} p_{i t}$ where $r_{i}$ is the initial beam radius. Because of the angle conservation, these two vectors are 2D orthogonal again after the transformation. Without a lack of generality, the $x$ axis can be assumed to go along the vector $\vec{x}_{1}$ both for the initial and the final states; this follows from symplecticity of the rotations. So the final states can be presented as

$$
\begin{aligned}
& \mathbf{x}_{1 f}=\left(r_{f}, p_{f r}, 0, p_{f t}\right), \\
& \mathbf{x}_{2 f}= \pm \tilde{\mathbf{x}}_{1 f} \equiv \pm\left(0,-p_{f t}, r_{f}, p_{f r}\right),
\end{aligned}
$$

with $M_{f}= \pm r_{f} p_{f t}$ as the final angular momentum.

In fact, the symplecticity condition (58) for a given vector $\mathbf{x}_{1}$ and arbitrary $\mathbf{x}_{2}$ is equivalent to the particular choice (59) and (60). Indeed, for a given $\mathbf{x}_{1}$, any $\mathbf{x}_{2}$ can be expanded over the two orthogonal vectors: $\mathbf{x}_{1}$ and its orthogonal counterpart $\tilde{\mathbf{x}}_{1}$. Then, the part of $\mathbf{x}_{2}$ parallel to $\mathbf{x}_{1}$ gives an identical zero for both sides of the symplecticity condition (58), while the component along $\tilde{\mathbf{x}}_{1}$ gives the same result as (59). 
Conservation of the skew-scalar product

$$
\left[\mathbf{x}_{1 i}, \mathbf{x}_{2 i}\right]=\left[\mathbf{x}_{1 f}, \mathbf{x}_{2 f}\right]
$$

for the orthogonal pair $\mathbf{x}_{1}, \mathbf{x}_{2}$ immediately yields $M_{i}=$ $\pm M_{f}$, as was to be shown.

Actually, the statement just proved means that the property of the canonical momentum conservation goes beyond the mapping (or Hamiltonian) invariance. For the invariant mappings, any initially symmetric state of beam transforms into a symmetric state again. It was shown above that the mapping invariance does not follow from the symmetry preservation for one beam state, a property which was referred to as the projective invariance. It was proved in fact that the mapping invariance is a somewhat surplus requirement for the momentum conservation; the projective invariance for a particular initial ensemble is sufficient to claim that every particle of this ensemble restores the value of its CAM as well. Note, however, that the sign of the final CAM is not fixed.

Turning back to the specific question at the beginning of this section, it can be concluded that there is no mapping, invariant or not, transforming a round but not properly magnetized (4) beam at the cathode into a calm round beam in the cooler. No transformation can change an absolute value of the canonical angular momentum of a particle without breaking the rotational symmetry of their ensemble.

\section{B. Canonical emittances and beam adapters}

Therefore, the generalized Busch's theorem asserts that a round electron beam at the cathode has to be properly magnetized. However, it says nothing about nonround beams at the cathode. In particular, what type of nonround, nonmagnetized beams can be transformed into a calm state in the cooling solenoid? By the definition, the drift emittance of a calm, or a magnetized, state is much higher than the cyclotron emittance. It seems rather obvious that for this beam there is a specific choice of canonical variables when the ratio between two independent canonical emittances at the initial state is equal to the ratio of the drift and cyclotron emittances in the cooler. Thus, to become magnetized in the cooler, the beam has to be described initially by the two canonical emittances of very different values. Obviously, a similar statement is related to a reverse transition. In particular, it can be expected that a flat beam $\varepsilon_{x} \gg \varepsilon_{y}$ can be injected into a solenoid with a proper optical adaptation to become a magnetized beam with solenoidal emittances $\varepsilon_{C}$ and $\varepsilon_{D}[10,21,22]$ having

$$
\frac{\varepsilon_{C}}{\varepsilon_{D}}=\frac{\bar{\rho}_{L}^{2}}{a^{2}}=\frac{\varepsilon_{y}}{\varepsilon_{x}} .
$$

Such schemes can be used in order to optimize the features of electron storage rings and recirculators as coolers for high-GeV hadron beams [9,23-25] and for other applications [26]. The transformation from the ribbon state in a free space into the magnetized state inside the solenoid looks promising for high-energy electron cooling projects $(\gamma=100-1000)$ where a natural flat shape of an electron beam in a storage ring can be utilized. However, it does not look as promising for the medium energy electron cooling where it would require a threadlike cathode with a too high aspect ratio $\left(\sigma_{y} / \sigma_{x}=\rho_{L}^{2} / a^{2}\right)$.

\section{FERMILAB ELECTRON COOLING PROJECT}

To increase Tevatron luminosity, Fermilab is developing a high energy electron cooling system to cool $8.9 \mathrm{GeV} / c$ antiprotons in the recycler ring [4]. A scheme of the electron transport proposed for this project incorporates many of the above ideas. This scheme is presented here as an example of how these ideas can be implemented.

The electron transport line employs an electrostatic accelerator Pelletron with the gun immersed into a longitudinal magnetic field. For the cathode radius of $2.5 \mathrm{~mm}$, the field of $600 \mathrm{G}$ on its surface was chosen to provide the magnetic flux sufficient to suppress the space charge drift motion inside the cooler (5). The magnetic field extends up to an end of the first acceleration section where it is already reduced to $200 \mathrm{G}$ while the electrons have $0.43 \mathrm{MeV}$ of the kinetic energy [see Eq. (7) and the estimations after it].

When the electron beam exits this field region, it continues to be accelerated in the Pelletron, having two focusing kicks by thin solenoid lenses during the acceleration. After that, the beam is to be delivered to the cooling section. This part of the transport line includes two $90^{\circ}$ bending blocks with solenoid lenses before and after every one of them. To deliver the beam from the accelerator to the cooling section, it must be turned in two different planes: first in the vertical and then in the horizontal. Each of the two

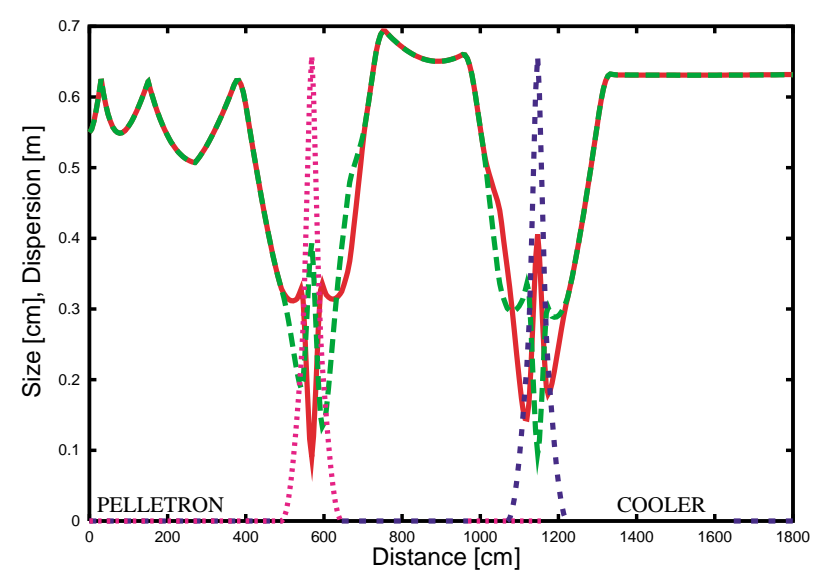

FIG. 3. (Color) Beam envelopes (green as vertical and red as horizontal) and the dispersion functions (magenta as vertical and blue as horizontal) for the Fermilab electron cooling project. The two bending blocks shown consist of two separated $45^{\circ}$ bends with a symmetric triplet in between and a couple of quads downstream/upstream of them. A major part of the cooler and the return line are not shown. 


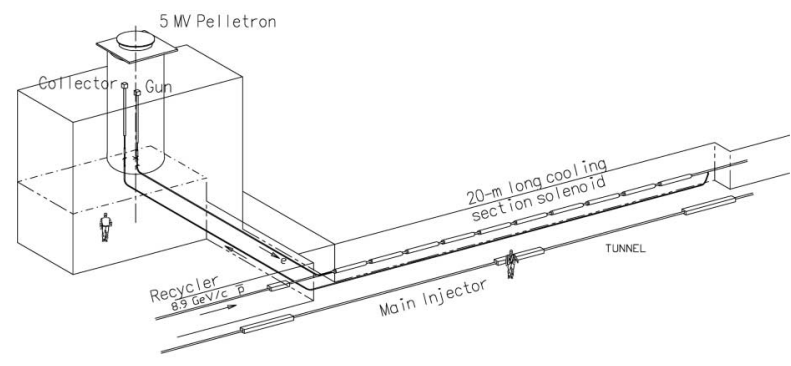

FIG. 4. Designed layout of the electron cooling beam line at Fermilab.

mirror-symmetric blocks consists of two $45^{\circ}$ bending magnets with a symmetric quadrupole triplet between them and two quadrupoles after (before) the resulting $90^{\circ}$ bend. This construction allows one to reach several goals.

First, it allows one to have zero dispersion downstream of the block, which is important both for the cooling conditions and for the electron beam stability. To eliminate dispersion, a $90^{\circ}$ bend has to be separated into two halves with a focusing element inserted in between. In principle, this central focusing element could be either a solenoid, a single quadrupole, or a symmetric triplet. The triplet is chosen because the required solenoid would be too heavy, while the single quadrupole would give too wide beam inside of the downstream dipole.

Second, this bending scheme provides an invariant mapping for the whole bending block (the mapping is block invariant). Beam parameters at the exit of the Pelletron cannot be current independent. Thus, tunable optical elements are necessary in the beam line for beam matching (4). To keep the line block invariant, these tunable elements can be either solenoids or invariant quadrupole blocks. Use of the solenoids looks more preferable for this purpose because technically it is easier to tune them. Finally, the beam must be small enough inside the dipoles and other elements to suppress nonlinear aberrations.

The two solenoids between the bending blocks allows one to have reasonable beam envelope for the second bending block and the matched beam radius at the entrance of the cooling section. The last solenoid upstream of the cooling section provides the zero radial divergence inside the cooler.

TABLE I. Electron cooling system parameters.

\begin{tabular}{lcc}
\hline \hline \multicolumn{1}{c}{ Parameter } & Value & Units \\
\hline Electron kinetic energy & 4.3 & $\mathrm{MeV}$ \\
Electron beam current & 0.5 & $\mathrm{~A}$ \\
Cathode radius & 2.5 & $\mathrm{~mm}$ \\
Cathode solenoid field & 600 & $\mathrm{G}$ \\
Cooling length & 20 & $\mathrm{~m}$ \\
Cooling solenoid field & 100 & $\mathrm{G}$ \\
Beam radius & 6.1 & $\mathrm{~mm}$ \\
Electron beam angles & $<100$ & $\mu \mathrm{rad}$ \\
\hline \hline
\end{tabular}

The electron transport simulations were done with the program OPTIM [27], which is an interactive Windows application allowing a visual optics design. The beam envelopes from the exit of the gun solenoid to the beginning of the cooling section are presented in Fig. 3.

Figure 4 shows the schematic layout of the system. Its main parameters are summarized in the Table I.

\section{SUMMARY}

The main purpose of this paper was to show how an electron beam for relativistic electron cooling can be transported by means of isolated focusing elements and bends, without any excitation of the cyclotron motion in the cooling solenoid. The introduced concepts of the angular momentum dominated beam and the effective beta function showed the region of parameters where the lumped focusing can be used. For the beam lines consisting of the optically symmetric elements (local invariant lines), the two Courant-Snyder invariants were found and conditions for the beam matching between the cathode and the electron cooler were discussed.

For the general kind of beam lines, it was demonstrated that the beam matching can be formulated as uncoupling of the drift and the cyclotron canonic degrees of freedom under the beam transportation. For rotationally invariant mappings, it again leads to the same matching condition and temperature transformation as for the locally invariant case. The concept of a block-invariant line was introduced, the general form of the invariant matrices was discussed, and utility for the whole line that consists of invariant blocks was pointed out. It was shown that any transformation can be transmuted to an invariant one by means of three free quadrupoles.

The generalized Busch's theorem was extended to the whole class of linear Hamiltonian systems. It was pointed out that according to this theorem the identical matching condition is valid when any hydrodynamic round beam is transformed into round beam again. Possibilities were discussed to use initially flat beams converted into round in the cooler. A general condition on the initial beam state was formulated for having a magnetized beam in the cooler. As an example of application of the developed ideas, the electron transport scheme for the Fermilab cooling project was presented.

The described methods of matching between the solenoid of the cooling region and the rest of the electron beam track (with a round or flat beam) can also serve as guiding principles for a design of recirculators and storage rings for high energy electron cooling.

\section{ACKNOWLEDGMENTS}

The authors are indebted to Valeri Lebedev (Jefferson Laboratory) for his code OPTIM and for numerous fruitful discussions. We also thank Jim MacLachlan (FNAL) for his permanent interest in this issue. One of the 
authors (Ya.D.) expresses gratitude to Reinhard Brinkmann (DESY) and Peter Schwandt (IUCF) for giving him the opportunity to collaborate in the development of relativistic electron cooling projects.

[1] G. I. Budker, At. Energ. 22, 346-348 (1967) [Sov. J. At. Energy 22, 438 (1967)].

[2] G. I. Budker and A. N. Skrinsky, Sov. Phys. Usp. 21, 277 (1978).

[3] I. N. Meshkov, Phys. Part. Nucl. 25, 631 (1994).

[4] S. Nagaitsev, et al., in Proceedings of the Particle Accelerator Conference, New York (PAC'99) (IEEE, Piscataway, NJ, 1999), pp. 521-523; S. Nagaitsev et al., in Proceedings of ECOOL99 [Nucl. Instrum. Methods Phys. Res., Sect. A 441, 241 (2000)].

[5] P. Wesolowski, K. Balewski, R. Brinkmann, Y. Derbenev, and K. Floettmann, Nucl. Instrum. Methods Phys. Res., Sect. A 441, 281 (2000).

[6] N. Dikansky et al., Part. Accel. 7, 197 (1976).

[7] N. Dikansky, S. Nagaitsev, and V. Parkhomchuk, Fermilab Report No. FERMILAB-TM-1998-H, 1996.

[8] N. Dikansky et al., in Proceedings of the Particle Accelerator Conference, Vancouver, Canada, 1997 (PAC'97) (IEEE, Piscataway, NJ, 1997), p. 1795.

[9] A. Burov, V. Danilov, Ya. Derbenev, and P. Colestock, in Proceedings of ECOOL99 (Ref. [4]), p. 271.

[10] Y. Derbenev, in Proceedings of ECOOL99 (Ref. [4]), p. 223.

[11] L. D. Landau and E. M. Lifshits, The Classical Theory of Fields (Pergamon Press, Addison-Wesley, New York, 1987).
[12] M. Reiser, Theory and Design of Charged Particle Beams (Wiley \& Sons, New York, 1994).

[13] A. Chao and M. Tigner, Handbook of Accelerator Physics and Engineering (World Scientific, Singapore, 1998), p. 49.

[14] H. Goldstein, Classical Mechanics (Addison-Wesley, Reading, MA, 1980), 2nd ed.

[15] V. I. Arnold, Mathematical Methods of Classical Mechanics (Springer-Verlag, Berlin, 1984).

[16] S. Nagaitsev and A. Shemyakin, Fermilab Report No. Fermilab-TM-2107, 2000.

[17] E. Pozdeev (private communication).

[18] E. A. Perevedentsev (private communication).

[19] V. V. Danilov and V. D. Shiltsev, Fermilab Report No. FERMILAB-FN-655, 1997.

[20] P. T. Kirstein, G. S. Kino, and W. E. Waters, Space Charge Flow (McGraw-Hill, New York, 1967).

[21] Y. Derbenev, University of Michigan Report No. UM HE 98-04, 1998.

[22] A. Burov and V. Danilov, Fermilab Report No. FERMILAB-TM-2043, 1998.

[23] A. Burov, P. Colestock, V. Danilov, Ya. Derbenev, and S. Y. Lee (private communication).

[24] K. Balewski, R. Brinkmann, Y. Derbenev, K. Floettmann, and P. Wesolowski in Proceedings of ECOOL99 (Ref. [4]), p. 274.

[25] P. Schwandt, in Proceedings of the 16th RCNP Osaka International Symposium on Multi-GeV High Performance Accelerators and Related Technology (World Scientific, Singapore, 1997), p. 185.

[26] R. Brinkmann, Y. Derbenev, and K. Floettmann, Report No. TESLA 99-09, DESY, 1999.

[27] V. A. Lebedev and S. A. Bogacz, e-print JLAB-ACC-99-19. 